PROCEEDINGS OF THE

AMERICAN MATHEMATICAL SOCIETY

Volume 42, Number 1, January 1974

\title{
A CHARACTERIZATION OF THE NONDIFFERENTIABUITY SET OF THE CANTOR FUNCTION
}

\author{
J. A. EIDSWICK
}

\begin{abstract}
The points of nondifferentiability of the Cantor ternary function are characterized in terms of the spacing of 0 's and 2's in ternary expansions. One consequence is an illustration of Morse's intermediate-value theorem for Dini derivates.
\end{abstract}

1. The characterization. Let $\theta$ be the Cantor ternary function, $T$ the Cantor ternary set (see, e.g., [3, p. 213]). In view of the fact that $\theta$ does all of its "climbing" on $T$, a nowhere dense set of measure zero, and because of the symmetry of its construction, one might think that both onesided derivatives exist everywhere on $[0,1]$ as $+\infty$ or 0 . A type of intermediate-value theorem by A. Morse [2] shows that this is not the case. In fact $\left[2\right.$, p. 130], for each $\lambda \geqq 0,\left\{x: \theta_{+}(x)=\lambda\right\}$ has the power of the continuum. On the other hand, it is easy to show that the upper derivates are always $+\infty$ or 0 . Also [6, p. 278], since $\theta$ satisfies Banach's $\left(T_{1}\right)$ condition, the set $\theta\left(N^{*}\right)$ has measure zero where $N^{*}$ is the set of points at which $\theta$ fails to have a derivative, finite or infinite. In other words, $\theta^{\prime}(t)=+\infty$ at most points $t$ of $T$ in the sense of measure (the measure on $T$ induced by $\theta$ ). Finally, it follows from Morse's work [2, p. 128] that $\theta(Z)$ is residual in $[0,1]$ where $Z$ is the set of points at which the lower derivates vanish. Consequently, $\theta_{-}(t)=0=\theta_{+}(t)$ at most points $t$ of $T$ in the sense of category.

The following theorem (i) characterizes the points $t$ of $T$ at which $\theta^{\prime}(t)=+\infty$, (ii) characterizes the points $t$ of $T$ at which $\theta_{-}(t)=0=\theta_{+}(t)$, (iii) leads to a concrete illustration of Morse's theorem, (iv) gives a clear picture of the smallness of $\theta\left(N^{*}\right)$, and (v) leads to some interesting questions of a more general nature.

THEOREM 1. Let $t$ be a point of $T$ which is not an endpoint of a complementary interval, let $z(n)$ denote the position of the nth zero in its ternary expansion, and let $\lambda=\lim \inf 3^{z(n)} / 2^{z(n+1)}$. Then $\lambda \leqq \theta_{+}(c) \leqq 2 \lambda$. Furthermore, if $z(n+1)-z(n) \rightarrow \infty$, then $\theta_{+}(t)=\lambda$.

Received by the editors January 30, 1973.

AMS (MOS) subject classifications (1970). Primary 26A27, 26A30; Secondary $10 \mathrm{~F} 20$.

Key words and phrases. Dini derivates, normal numbers, Diophantine approximation. 
Likewise, if $t(n)$ denotes the position of the nth two in the expansion of $t$ and $\mu=\lim \inf 3^{t(n)} / 2^{t(n+1)}$, then $\mu \leqq \theta_{-}(t) \leqq 2 \mu$, equality holding on the right if $t(n+1)-t(n) \rightarrow \infty$.

ProOf. Let $t=t_{1} t_{2} \cdots$ be the ternary expansion of a point in $T$, not an endpoint of a complementary interval, and define $d_{n}=. t_{1} \cdots t_{z(n)-1} 1$, $e_{n}=. t_{1} \cdots t_{z(n)-1} 2$. The right-hand derivatives of $\theta$ at $t$ are completely determined by the "plateaus" of $\theta$ corresponding to the intervals $\left[d_{n}, e_{n}\right]$. To see this, observe that for $0<h_{n}<d_{n-1}-e_{n}=3^{-z(n)}$, we have $\theta\left(e_{n}+h_{n}\right)=$ $\theta\left(e_{n}\right)+\theta\left(h_{n}\right)$ and since $\theta^{\prime}(0)=+\infty$, it follows that

$$
\theta_{+}(t)=\liminf _{n \rightarrow \infty} Q_{n}
$$

where $Q_{n}=\left(\theta\left(e_{n}\right)-\theta(t)\right) /\left(e_{n}-t\right)$.

A straightforward calculation shows that

$$
Q_{n}=\frac{3^{z(n)}}{2^{z(n+1)}} \frac{\sum_{1}^{\infty} 2^{-(z(n+k)-z(n+1))}}{1+2 \sum_{1}^{\infty} 3^{-(z(n+k)-z(n))}}
$$

and, by elementary estimates,

$$
\left(1+3^{1-q}\right)^{-1} \cdot 3^{z(n)} / 2^{z(n+1)} \leqq Q_{n} \leqq 2 \cdot 3^{z(n)} / 2^{z(n+1)}
$$

where $q=z(n+1)-z(n)$. These inequalities imply that $\lambda \leqq \lim$ inf $Q_{n} \leqq 2 \lambda$. (Proof. Let $0<\varepsilon<1$. Then $Q_{n} \geqq(1-\varepsilon) 3^{z(n)} / 2^{z(n+1)}$ if $q \geqq \varepsilon^{-1}$ and $Q_{n} \geqq$ $\left(3+3^{1 / \varepsilon}\right)^{-1}(3 / 2)^{2(n+1)}$ if $q<\varepsilon^{-1}$. Therefore, $\lim \inf Q_{n} \geqq(1-\varepsilon) \lambda$ and by the arbitrariness of $\varepsilon, \lambda \leqq \lim \inf Q_{n}$. The other inequality is obvious.) Hence, by (1), $\lambda \leqq \theta_{+}(t) \leqq 2 \lambda$. Also, it is clear from (2) that if $z(n+1)-z(n) \rightarrow \infty$, then $\theta_{+}(t)=\lambda$.

The second statement of the theorem is proved in a similar way.

The following two corollaries are immediate.

COROLLARY 1. In the notation of Theorem $1, \theta^{\prime}(t)=+\infty$ if and only if $\lambda=\mu=+\infty$.

COROLlaRY 2. In the notation of Theorem $1, \theta_{-}(t)=0=\theta_{+}(t)$ if and only if $\lambda=\mu=0$.

A number $x \in[0,1]$ is called simply normal to base 2 if $n / z(n) \rightarrow \frac{1}{2}$ where $z(n)$ denotes the position of the $n$th zero in the binary expansion of $x$. For the general theory of normal numbers, see [5]. It is known [5, p. 103] that the set of numbers which are not simply normal to base 2 (indeed, a considerably larger class) has measure zero. We will use this fact to show that $\theta\left(N^{*}\right)$ has measure zero. Let $x=\theta(t)$ where $t$ is a point of $T$ which is not an endpoint of a complementary interval, and suppose $\theta^{\prime}(t) \neq+\infty$. 
By Corollary 1 , either $\lambda$ or $\mu$, say $\lambda$, is finite. It follows that

$$
\lim \inf [z(n) \xi-z(n+1)]<+\infty,
$$

where $\xi=\log 3 / \log 2$. Hence, $\{z(n)\}$ has a subsequence $\left\{z\left(n_{k}\right)\right\}$ such that $z\left(n_{k}+1\right)-z\left(n_{k}\right) \geqq \frac{1}{2} z\left(n_{k}\right)$. Thus $x$ cannot be simply normal to base 2 . We have proved the following result.

COROllary 3. No point of $\theta\left(N^{*}\right)$. is simply normal to base 2. In particular, $\theta\left(N^{*}\right)$ has measure zero.

The conclusion of Theorem 1 is the best possible. In fact, for any $\lambda>0$, there exists a point $t=t(\lambda)$ such that $\theta_{+}(t)=2 \lambda$ and

$$
\lim \inf 3^{z(n)} / 2^{z(n+1)}=\lambda .
$$

Such a point is given by

$$
t=\sum_{n=1}^{\infty}\left(\frac{2}{3^{a_{n}+1}}+\cdots+\frac{2}{3^{b_{n}-1}}\right)
$$

where $\left\{a_{n}\right\}$ and $\left\{b_{n}\right\}$ are increasing sequences of positive integers such that (i) $a_{n} \xi-b_{n} \rightarrow \log \lambda / \log 2$ where $\xi=\log 3 / \log 2$, (ii) $b_{n}-a_{n} \geqq 2$ for all $n$, and (iii) $a_{n+1}-b_{n} \rightarrow \infty$. (The existence of such sequences follows from the fact that the numbers $\xi, 2 \xi, \cdots$ are uniformly distributed modulo 1 . See $[5$, p. 72].)

2. An illustration of Morse's theorem. Actually we illustrate a property more general than that considered by Morse:

THEOREM 2. For each $\mu \geqq 0$ and $\lambda \geqq 0$, the set

$$
T_{\mu \lambda}=\left\{t \in T: \theta_{-}(t)=\mu \text { and } \theta_{+}(t)=\lambda\right\}
$$

has the power of the continuum.

ProOf. Let $\mu_{0}=\log \mu / \log 2, \lambda_{0}=\log \lambda / \log 2$. Let $\left\{a_{n}\right\}$ and $\left\{b_{n}\right\}$ be increasing sequences of positive integers such that $a_{2 n} \xi-b_{2 n} \rightarrow \lambda_{0}$ and $a_{2 n+1} \xi-b_{2 n+1} \rightarrow \mu_{0}$ where $\xi=\log 3 / \log 2$. We may assume that $a_{n}<b_{n}$, $a_{n+1}-b_{n} \geqq 4 n$, and $b_{n} \geqq 3 n$ for all $n$. Let $P_{n}=\left[b_{n 0}, b_{n 1}, \cdots, b_{n r_{n}}\right]$ be an integral partition of $\left[b_{n}, a_{n+1}\right]$ with $n \leqq\left\|P_{n}\right\| \leqq 2 n$. Define $t_{m}=0$ if $m=a_{2 n}, b_{2 n}, b_{2 n, 1}, \cdots, b_{2 n, \tau_{2 n}-1}$ or if $b_{2 n+1, i-1}<m<b_{2 n+1, i}, i=1, \cdots$, $r_{2 n+1}$, and define $t_{m}=2$ for all other values of $m$. Then it follows from Theorem 1 and formula (2) of its proof that $t=\sum t_{m} / 3^{m} \in T_{\mu \lambda}$. Moreover, since there are at least two choices for each $P_{n}$, the set of all such $t$ has the cardinality of the continuum. 
3. Unsolved problems. A natural question to ask is: Does there exist an increasing sequence $\{z(n)\}$ of positive integers such that $\lim 3^{z(n)} / 2^{z(n+1)}=$ 1 (say)? More generally, for which numbers $\xi$ and $\eta$ does there exist an increasing sequence $\{z(n)\}$ of positive integers such that $\lim [z(n) \xi-z(n+1)]=\eta$ ? The problem is reminiscent of Diophantine approximation problems [4] but the literature does not seem to deal with this particular brand of "continued fractions". The classical theory of continued fractions shows that solutions exist. For example [1, p. 163], if $\xi=\frac{1}{2}(1+\sqrt{ } 5)$ and $\eta=0$, then the sequence of Fibonacci numbers is a solution.

A second problem arises from Theorem 2: Does Morse's theorem generalize? That is, if $f$ is continuous, $\left\{x: f_{-}(x) \leqq \mu\right.$ and $\left.f_{+}(x) \leqq \lambda\right\}$ is dense, and $\left\{x: f_{-}(x)>\mu\right.$ and $\left.f_{+}(x)>\lambda\right\}$ is nonempty, then is it true that $\left\{x: f_{-}(x)=\mu\right.$ and $\left.f_{+}(x)=\lambda\right\}$ has the cardinality of the continuum? A modification of the category argument used by Morse gives an affirmative answer when $\lambda=\mu$, but apparently different methods are needed to handle the general case.

\section{REFERENCES}

1. G. H. Hardy and E. M. Wright, An introduction to the theory of numbers, 4th ed., Oxford Univ. Press, London, 1960.

2. A. Morse, Dini derivatives of continuous functions, Proc. Amer. Math. Soc. 5 (1954), 126-130. MR 15, 610.

3. I. P. Natanson, Theory of functions of a real variable, 2 nd rev. ed., GITTL, Moscow, 1957; English transl., Ungar, New York, 1961. MR 26 \#6309.

4. I. Niven, Diophantine approximations, The Ninth Annual Series of Earle Raymond Hedrick Lectures of the Math. Assoc. Amer., Interscience Tracts in Pure and Appl. Math., no. 14, Interscience, New York, 1963. MR 26 \#6120.

5. - Irrational numbers, Carus Math. Monographs, no. 11, Math. Assoc. Amer., Wiley, New York, 1956. MR 18, 195.

6. S. Saks, Theory of the integral, 2nd rev. ed., Monografie Mat., vol. VII, PWN, Warsaw, 1937.

Department of Mathematics, University of Nebraska, Lincoln, Nebraska 68508 